\title{
Conservation-Based Modeling and Boundary Control of Congestion with an Application to Traffic Management in Center City Philadelphia
}

\author{
Xun Liu and Hossein Rastgoftar ${ }^{2}$
}

\begin{abstract}
This paper develops a conservation-based approach to model traffic dynamics and alleviate traffic congestion in a network of interconnected roads (NOIR). We generate a NOIR by using the Simulation of Urban Mobility (SUMO) software based on the real street map of Philadelphia Center City. The NOIR is then represented by a directed graph with nodes identifying distinct streets in the Center City area. By classifying the streets as inlets, outlets, and interior nodes, the model predictive control (MPC) method is applied to alleviate the network traffic congestion by optimizing the traffic inflow and outflow across the boundary of the NOIR with consideration of the inner traffic dynamics as a stochastic process. The proposed boundary control problem is defined as a quadratic programming problem with constraints imposing the feasibility of traffic coordination, and a cost function defined based on the traffic density across the NOIR.
\end{abstract}

\section{INTRODUCTION}

In the process of urbanization and the rapid popularization of private vehicles, the problem of urban traffic congestion has become more and more prominent and produced numerous negative impacts on economy [1], [2] and environment [3], [4]. Traffic congestion can destroy the urban environment and ecology. Due to the low-speed driving conditions, the emission of greenhouse gas, noxious gas and noise will increase, and that will badly affect human health [5].

Over the past decades, a large number of scholars have developed prediction, control, and optimization methods to solve the challenges of traffic congestion in urban areas. Ref. [6] offers an integration of fuzzy rule-based systems and the genetic algorithms to model and predict the traffic coordination. Refs. [7] and [8] develop the traffic predictive approaches by relying on driver behavior and bus driving intervals. With the rapid development of V2X and autonomous driving technology, floating car data (FCD) technology has been widely used to estimate the traffic state [9], [10].

Researchers have also developed different model-based and model-free approaches to obtain dynamics of traffic coordination and control congestion. The model-based macroscopic fundamental diagram (MFD), whose applicability for urban traffic is experimentally verified in [11], is an efficient tool to obtain dynamics of an urban traffic network. Ref. [12] applies the MFD model to evaluate the traffic accumulation amount, and estimate the traffic state. Refs. [13], [14] integrate MFD with perimeter control to improve the mobility of a traffic network. Moreover, Refs. [15][19] apply the cell transmission model (CTM) method to

The authors are with the Department of Mechanical Engineering at Villanova University, Villanova, PA 19085, USA \{xliu8, hossein.rastgoftar\}@villanova.edu enhance the efficiency and accuracy of the network modeling by partitioning the traffic network into homogeneous road elements. Recently, with the improvement of computing capacity and the development of AI technology, reinforcement learning (RL) method has attracted more and more attention. Ref. [20] presents an overview of the recently-developed RL algorithms in the area of adaptive traffic signal control. In Refs. [21]-[24], researchers integrate the model-free methods with RL approaches to optimally plan the functionalities of traffic signals. The model predictive control (MPC) is another commonly used tool for controlling the traffic dynamics in urban networks. Refs. [14], [13], [25], [26] and [27] apply the MPC approach to assign optimal boundary control variables. Ref. [28] integrates the MPC and mixed-integer linear programming (MILP) to manage the complexity of traffic coordination optimization.

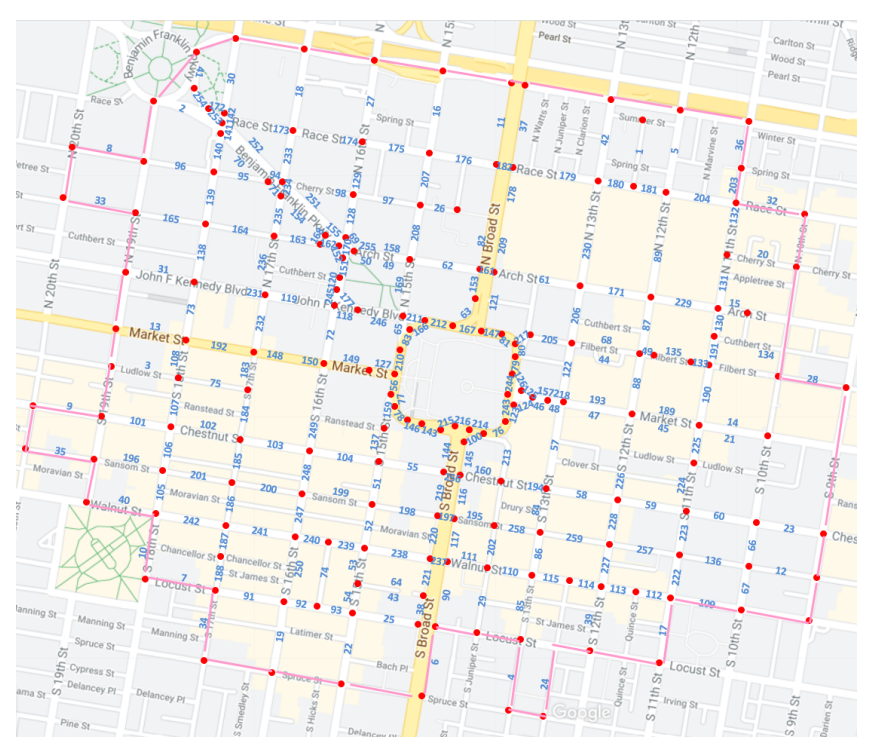

Fig. 1: Example NOIR: Center City, Philadelphia

This paper offers an integration of mass conservation law and MPC-based boundary control to obtain the traffic dynamics and alleviate the traffic congestion. We use the Simulation of Urban Mobility (SUMO) software to convert the real street map data into a directed graph representing a network of inter-connected roads (NOIR). While we previously modeled traffic inner dynamics as a time-invariant stochastic process in Refs. [25]-[27], this paper applies the mass conservation law to model the traffic inner dynamics as a non-stationary stochastic process and obtain the traffic feasibility conditions at the interior nodes. Compared to Refs. [25]-[27] that control the congestion only through the inlet 
boundary nodes, we apply the MPC to control the boundary inflow through the NOIR inlets, and the boundary outflow through the NOIR outlets. For the case study, we use the proposed model and control approach to evaluate congestion management in a certain area of Center City Philadelphia with the map shown in Fig. 1.

This paper is arranged in the following structure: Section III explains the preliminary notions of graph theory. The problem statement is presented in Section III and followed by obtaining the traffic network dynamics and providing the feasibility conditions in Section IV Section V presents the boundary control approach based on the MPC method to control the traffic congestion. Then, the simulation results are reported in Section $\mathrm{VI}$ and followed by the conclusion in Section VII

\section{Preliminary Notions of Graph Theory}

In this paper, an NOIR is represented by graph $\mathcal{G}(\mathcal{V}, \mathcal{E})$ where $\mathcal{V}$ and $\mathcal{E} \subset \mathcal{V} \times \mathcal{V}$ define nodes and edges of graph $\mathcal{G}$, respectively. We use $i \in \mathcal{V}$ to represent a road element in the NOIR. Note that all of the road elements partitioned and generated by SUMO in the NOIR are unidirectional. The bidirectional road is presented by two parallel one-way road elements (See Fig.1). Also, since we are more interested in the boundary traffic dynamics, the traffic light controller is not in consideration in this paper. Edge $(i, j) \in \mathcal{E}$ represents a directed connection from road element $i \in \mathcal{V}$ to road element $j \in \mathcal{V}$.

Set $\mathcal{V}$ can be partitioned as $\mathcal{V}=\mathcal{V}_{\text {in }} \cup \mathcal{V}_{\text {out }} \cup \mathcal{V}_{I}$, where subsets $\mathcal{V}_{\text {in }}=\left\{1, \cdots, N_{\text {in }}\right\}, \mathcal{V}_{\text {out }}=\left\{N_{\text {in }}+1, \cdots, N_{\text {out }}\right\}$, and $\mathcal{V}_{I}=\left\{N_{\text {out }}+1, \cdots, N\right\}$ define the index numbers of inlets, outlets, and interior road elements respectively. For every road element $i \in \mathcal{V}$, sets

$$
\begin{aligned}
\mathcal{I}_{i} & =\{j:(j, i) \in \mathcal{E}\}, \\
O_{i} & =\{j:(i, j) \in \mathcal{E}\}
\end{aligned}
$$

define in-neighbors and out-neighbors. Traffic is directed from in-neighbor $j \in \mathcal{I}_{i}$ towards $i \in \mathcal{V} \backslash \mathcal{V}_{i n}$, or it is directed from $i \in \mathcal{V} \backslash \mathcal{V}_{\text {out }}$ towards out-neighbor $j \in O_{i}$.

\section{Problem Statement}

We implement the mass-conservation law to obtain traffic dynamics at every road element $i \in \mathcal{V}$. Let $s_{i}[k]$ denote the external flow, and $\rho_{i}[k], y_{i}[k]$, and $z_{i}[k]$ denote traffic density, network inflow, and network outflow of road $i \in \mathcal{V}$, respectively. Then, traffic dynamics at road element $i \in \mathcal{V}$ can be defined by

$$
\rho_{i}[k+1]=\rho_{i}[k]+y_{i}[k]-z_{i}[k]+s_{i}[k]
$$

where $k=0,1,2, \cdots$ denotes the discrete sampling time.

External flow $s_{i}[k]$ quantifies the traffic inflow entering the NOIR through inlet road element $i \in \mathcal{V}_{i n}$, or the traffic outflow leaving the NOIR through outlet road element $i \in$ $\mathcal{V}_{\text {out }}$ within time interval $\left[t_{k}, t_{k+1}\right]$. We define $s_{i}[k]$ as follows:

$$
s_{i}[k]= \begin{cases}u_{i}[k] \geq 0 & i \in \mathcal{V}_{\text {in }} \\ -v_{i}[k] \leq 0 & i \in \mathcal{V}_{\text {out }} . \\ 0 & i \in \mathcal{V}_{I}\end{cases}
$$

Network inflow $y_{i}[k]$ and network outflow $z_{i}[k]$ are given by

$$
\begin{aligned}
y_{i}[k] & = \begin{cases}0 & i \in \mathcal{V}_{\text {in }} \\
v_{i}[k] & i \in \mathcal{V}_{\text {out }}, \\
\sum_{j \in \mathcal{I}_{i}} q_{i, j}[k] z_{j}[k] & i \in \mathcal{V}_{I}\end{cases} \\
z_{i}[k] & = \begin{cases}u_{i}[k] & i \in \mathcal{V}_{\text {in }} \\
0 & i \in \mathcal{V}_{\text {out }}, \\
p_{i}[k] \rho_{i}[k] & i \in \mathcal{V}_{I}\end{cases}
\end{aligned}
$$

where

$$
p_{i}[k]= \begin{cases}1 & \text { If } i \in \mathcal{V}_{\text {in }} \cup \mathcal{V}_{\text {out }} \\ 0 & \text { If } i \in \mathcal{V}_{I} \text { and } \rho_{i}=0 \\ \frac{z_{i}[k]}{\rho_{i}[k]} & \text { If } i \in \mathcal{V}_{I} \text { and } \rho_{i} \neq 0\end{cases}
$$

is the outflow probability of road element $i \in \mathcal{V}_{I}$ at discrete time $k, q_{i, j} \in[0,1]$ is the fraction of outflow traffic directed from $j \in \mathcal{V} \backslash \mathcal{V}_{\text {out }}$ to $i \in O_{j}$ at every discrete time $k$, and

$$
\sum_{i \in O_{j}} q_{i, j}=1
$$

at every interior road $i \in \mathcal{V}_{I}$.

Given the above problem setting, the main purpose of this paper is to alleviate the traffic congestion by assigning the optimal external flow $s_{i}[k]$. Assuming $p_{i}[k]$ and $q_{i, j}[k]$ are known at every interior road $i \in \mathcal{V}_{I}$, the external flow is determined by solving a quadratic programming problem with cost function

$$
\mathrm{C}=\frac{1}{2} \sum_{l=0}^{N_{\tau}-1}\left(\sum_{i \in \mathcal{V}_{\text {in }}} u_{i}^{2}[k+l]+\sum_{j \in \mathcal{V}_{\text {out }}} v_{j}^{2}[k+l]\right)
$$

and the following inequality and equality constraints:

$$
\begin{gathered}
\bigwedge_{l=0}^{N_{\tau}-1} \bigwedge_{i \in \mathcal{V}_{\text {in }}}\left(u_{i}[k+l] \geq 0\right), \\
\bigwedge_{l=0}^{N_{\tau}-1}\left(v_{j}[k+l] \geq 0\right), \\
\bigwedge_{l=0}^{N_{\tau}-1}\left(\bigwedge_{i=\mathcal{V}_{\text {out }}}^{N_{\tau}-1} \bigwedge_{i \in \mathcal{V}_{\text {in }}} \bigwedge_{i \in \mathcal{V}_{I}}\left(\rho_{i}[k+l] \geq 0\right),\right. \\
\left.\bigwedge_{i \in \mathcal{V}_{I}}[k+l]+\sum_{j \in \mathcal{V}_{\text {out }}} v_{j}[k+l]=d_{0}\right) .
\end{gathered}
$$

Note that $N_{\tau}<\infty$ is the time horizon length and $\rho_{i, \max }$ is the maximum number of vehicles that can be accommodated at road element $i \in \mathcal{V}_{I}$. Constraint Eqs. 8a and $8 \mathrm{~b}$ ensure that the traffic back-flow is avoided at every inlet or outlet 
road element. Constraint $8 \mathrm{c}$ ensures that the solution of the above optimization problem obtains a non-negative traffic density distribution across the NOIR. Constraint $8 \mathrm{~d}$ is imposed to avoid the traffic over-saturation. Assuming the demand for entering and leaving the NOIR is sufficiently high, equality constraint $8 \mathrm{e}$ ensures that $d_{0}$ cars can cross the border of the NOIR at every discrete time $k$.

\section{TRAFFIC NETWORK DYNAMiCS}

By substituting (3) and (4) in (2), the traffic dynamics at road element $i \in \mathcal{V}$ simplifies to

$$
\begin{gathered}
\forall i \in \mathcal{V}_{\text {in }} \bigcup \mathcal{V}_{\text {out }}, \quad \rho_{i}[k+1]=\rho_{i}[k] \\
\forall i \in \mathcal{V}_{I}, \quad \rho_{i}[k+1]=\left(1-p_{i}[k]\right) \rho_{i}[k]+\sum_{j \in I_{i}} q_{i, j}[k] p_{j}[k] \rho_{j}[k] .
\end{gathered}
$$

Eq. (9a) shows that the traffic density remains constant at inlet and outlet road elements. Thus, traffic dynamics are only defined for the interior road elements.

Assumption 1. Discrete time $k$ represents the time interval $\left[t_{k}, t_{k+1}\right)$ where the time increment $\Delta T=t_{k+1}-t_{k}$ is assumed to be constant for $k=0,1,2, \cdots$. We choose a sufficientlysmall time increment $\Delta T$ such that

$$
p_{i}[k]=\frac{z_{i}[k]}{\rho_{i}[k]} \in[0,1)
$$

at every road element $i \in \mathcal{V}_{I}$ and every discrete time $k$, if $\rho_{i}[k] \neq 0$. Note that $p_{i}[k]=0$, if $\rho_{i}[k]=0$ over the time interval $t \in\left[t_{k}, t_{k+1}\right)$ (See Eq. (5)).

To obtain the traffic network dynamics, we define state vector $\mathbf{x}=\left[\rho_{N_{\text {out }}+1} \cdots \rho_{N}\right]^{\top} \in \mathbb{R}^{\left(N-N_{\text {out }}\right) \times 1}$, NOIR inflow vector $\mathbf{y}=\left[y_{N_{\text {out }}+1} \cdots y_{N}\right]^{\top} \in \mathbb{R}^{\left(N-N_{\text {out }}\right) \times 1}$, NOIR outflow vector $\mathbf{z}=\left[z_{N_{\text {out }}+1} \cdots z_{N}\right]^{\top} \in \mathbb{R}^{\left(N-N_{\text {out }}\right) \times 1}$, outflow probability matrix $\mathbf{P}=\operatorname{diag}\left(p_{N_{\text {out }}+1}, \cdots, p_{N}\right) \in \mathbb{R}^{\left(N-N_{\text {out }}\right) \times\left(N-N_{\text {out }}\right)}$, and tendency probability matrix $\mathbf{Q}=\left[Q_{i j}\right]$ with $(i, j)$ entry

$$
Q_{i j}[k]=q_{i+N_{\text {out }}, j+N_{\text {out }}}[k] \text {. }
$$

Note that $q_{i+N_{\text {out }}, j+N_{\text {out }}}[k]$ is the fraction of outflow of road $\left(j+N_{\text {out }}\right) \in \mathcal{V}_{I}$ directed towards $\left(i+N_{\text {out }}\right) \in \mathcal{V}_{I}$.

Per Eq. 4a, the NOIR inflow vector $\mathbf{y}$ and outflow vector $\mathbf{z}$ can be related to $\mathbf{x}$ by

$$
\begin{aligned}
& \mathbf{y}[k]=\mathbf{Q}[k] \mathbf{P}[k] \mathbf{x}[k], \\
& \mathbf{z}[k]=\mathbf{P}[k] \mathbf{x}[k] .
\end{aligned}
$$

If Eq. 9b is applied to model traffic coordination at every interior node $i \in \mathcal{V}_{I}$, the traffic network dynamics become

$$
\mathbf{x}[k+1]=\mathbf{A}[k] \mathbf{x}[k]+\mathbf{B}[k] \mathbf{s}[k],
$$

where $\mathbf{s}[k]=\left[s_{i}[k]\right] \in \mathbb{R}^{N_{\text {out }} \times 1}, \quad \mathbf{B}[k]=\left[b_{i j}[k]\right] \in$ $\mathbb{R}^{\left(N-N_{\text {out }}\right) \times N_{\text {out }}, \mathbf{A}[k] \in \mathbb{R}^{\left(N-N_{\text {out }}\right) \times\left(N-N_{\text {out }}\right)} \text { are obtained as }}$ follows:

$$
\begin{gathered}
s_{i}[k]=\left\{\begin{array}{l}
u_{i}[k], \quad \text { If } i \in \mathcal{V}_{\text {in }}=\left\{1, \cdots, N_{\text {in }}\right\} \\
v_{i}[k], \quad \text { If } i \in \mathcal{V}_{\text {out }}=\left\{N_{\text {in }}+1, \cdots, N_{\text {out }}\right\}
\end{array},\right. \\
b_{i j}[k]=\left\{\begin{array}{ll}
1 & j \in \mathcal{I}_{i+N_{\text {out }}} \\
-1 & j \in \mathcal{O}_{i+N_{\text {out }}}
\end{array},\right. \\
\mathbf{A}[k]=\mathbf{I}-\mathbf{P}[k]+\mathbf{Q}[k] \mathbf{P}[k] .
\end{gathered}
$$

Theorem 1. Assume graph $\mathcal{G}(\mathcal{V}, \mathcal{E})$ holds the following properties:

1) Traffic inflow directs from every inlet boundary road element towards an interior road element.

2) There is at least one directed path from every interior node to an outlet node.

3) Graph $\mathcal{G}$ contains no isolated node.

4) No inlet boundary road element is directly connected to an outlet boundary road element.

Then, the traffic network dynamics (13) is BIBO stable.

Proof: If assumptions of Theorem 1 are satisfied, matrix $\mathbf{A}[k]$ holds the following properties at every discrete time $k$ :

1) All entries in matrix $\mathbf{A}[k]$ are non-negative.

2) Column $i$ of matrix $\mathbf{A}[k]$ sums up to 1 , if $O_{i+N_{\text {out }}} \cap \mathcal{V}_{\text {out }}=\emptyset$.

3) Elements of column $i$ of matrix $\mathbf{A}[k]$ sums up to a positive number in interval $(0,1)$, if $O_{i+N_{\text {out }}} \cap \mathcal{V}_{\text {out }} \neq$ $\emptyset$.

Thus, eigenvalues of matrix $\mathbf{A}[k]$ are all less than one at every discrete time $k$.

Per traffic dynamics (13), we can define

$$
\mathbf{x}[k+1]=\boldsymbol{\Theta}_{k}\left[\begin{array}{c}
\mathbf{x}[1] \\
\mathbf{B}[k] \mathbf{s}[1] \\
\vdots \\
\mathbf{B}[k] \mathbf{s}[k]
\end{array}\right],
$$

where

$$
\begin{aligned}
\boldsymbol{\Theta}_{k} & =\left[\begin{array}{llll}
\boldsymbol{\Gamma}_{k} & \cdots & \boldsymbol{\Gamma}_{1} & \boldsymbol{\Gamma}_{0}
\end{array}\right], \\
\boldsymbol{\Gamma}_{h} & =\prod_{j=k-h+1}^{k} \mathbf{A}[j],
\end{aligned}
$$

for $h=1, \cdots, k$, and $\boldsymbol{\Gamma}_{0}=\mathbf{I}_{N-N_{\text {out }}} \in \mathbb{R}^{\left(N-N_{\text {out }}\right) \times\left(N-N_{\text {out }}\right)}$ is an identity matrix. Because $\mathbf{x}[1]<\infty$, and $\mathbf{s}[k]$ is bounded at every discrete time $k$, we can write

$$
\begin{aligned}
\mathbf{x}[1] & \leq z_{\max } \mathbf{1}_{N-N_{\text {out }} \times 1}, \\
\mathbf{B}[k] \mathbf{s}[k] & \leq z_{\max } \mathbf{1}_{N-N_{\text {out }} \times 1},
\end{aligned}
$$

where $z_{\max }$ is bounded. If assumptions of Theorem 1 are satisfied, spectral radius $r$ of matrix $\boldsymbol{\Gamma}_{k}$ is less than 1 at every discrete time $k$. Therefore, we can write

$$
\begin{aligned}
\mathbf{x}^{\top}[k+1] \mathbf{x}[k+1] & \leq z_{\text {max }} \mathbf{1}_{N-N_{\text {out }} \times 1}^{\top}\left(\sum_{l=0}^{k} \sum_{h=0}^{k} \boldsymbol{\Gamma}_{l}^{\top} \boldsymbol{\Gamma}_{h}\right) z_{\text {max }} \mathbf{1}_{N-N_{\text {out }} \times 1} \\
& \leq z_{\text {max }}^{2}\left(N-N_{\text {out }}\right)\left(\sum_{l=0}^{\infty} r^{l}\right) \leq \frac{z_{\text {max }}^{2}\left(N-N_{\text {out }}\right)}{(1-r)}
\end{aligned}
$$

which implies that $\mathbf{x}^{\top}[k+1] \mathbf{x}[k+1]$ is bounded at every discrete time $k$, and thus the BIBO stability of traffic dynamics 13 is proven.

\section{TRAFFic Congestion CONTROL}

This paper applies the model predictive control (MPC) approach to control congestion through optimizing the boundary inflow and outflow. For the proposed MPC control, we 
use the linear time-varying dynamics 13 to predict traffic evolution within a future finite time horizon, and determine the optimal boundary external flow as a solution of the quadratic function subject to the inequality and equality constrains.

Given traffic dynamics (13) at discrete time, the following predictive model can be used to model traffic coordination within the next $N_{\tau}$ time steps:

$$
\mathbf{X}[k]=\mathbf{G}[k] \mathbf{x}[k]+\mathbf{H}[k] \mathbf{U}[k]
$$

where

$$
\begin{aligned}
& \mathbf{X}[k]=\left[\begin{array}{lll}
\mathbf{x}^{\top}[k+1] & \cdots & \mathbf{x}^{\top}\left[k+N_{\tau}\right]
\end{array}\right]^{\top} \in \mathbb{R}^{\left(N_{\tau} N\right) \times 1}, \\
& \mathbf{G}[k]=\left[\begin{array}{c}
\mathbf{A}[k] \\
\vdots \\
\mathbf{A}^{N_{\tau}[k]}
\end{array}\right] \in \mathbb{R}^{\left(N_{\tau} N\right) \times N} \\
& \mathbf{H}[k]=\left[\begin{array}{ccccc}
\mathbf{B}[k] & 0 & 0 & \cdots & 0 \\
\mathbf{A}[k] \mathbf{B}[k] & \mathbf{B}[k] & 0 & \cdots & 0 \\
\mathbf{A}^{2}[k] \mathbf{B}[k] & \mathbf{A}[k] \mathbf{B}[k] & \mathbf{B}[k] & \cdots & 0 \\
\vdots & \vdots & \vdots & & \vdots \\
\mathbf{A}^{N_{\tau}-1}[k] \mathbf{B}[k] & \mathbf{A}^{N_{\tau}-2}[k] \mathbf{B}[k] & \mathbf{A}^{N_{\tau}-3}[k] \mathbf{B}[k] & \cdots & \mathbf{B}[k]
\end{array}\right], \\
& \mathbf{x}[k]=\left[\begin{array}{lll}
\rho_{1}[k] & \cdots & \rho_{N}[k]
\end{array}\right]^{\top} \in \mathbb{R}^{N \times 1}, \\
& \mathbf{U}[k]=\left[\begin{array}{lll}
\mathbf{s}^{\top}[k] & \cdots & \mathbf{s}^{\top}\left[k+N_{\tau}-1\right]
\end{array}\right]^{\top} \in \mathbb{R}^{\left(N_{\tau} N_{\text {out }}\right) \times 1} \text {. }
\end{aligned}
$$

Now, we can rewrite the cost function (7) as

$$
\mathbf{C}=\frac{1}{2}\left(\mathbf{U}[k]^{\top} \mathbf{U}[k]\right) .
$$

By using the predictive traffic coordination model (19), we can also rewrite the constraint equations (8) as follows:

$$
\begin{gathered}
\mathbf{U}[k] \geq \mathbf{0}_{\left(N_{\tau} N_{\text {out }}\right) \times 1}, \\
\mathbf{G}[k] \mathbf{x}[k]+\mathbf{H}[k] \mathbf{U}[k] \leq \mathbf{1}_{N_{\tau} \times 1} \otimes \mathbf{x}_{\max }, \\
\mathbf{G}[k] \mathbf{x}[k]+\mathbf{H}[k] \mathbf{U}[k] \geq \mathbf{0}_{\left(N_{\tau} N\right) \times 1}, \\
\left(\mathbf{I}_{N_{\tau}} \otimes \mathbf{1}_{1 \times N_{\text {out }}}\right) \mathbf{U}[k]=d_{0} \mathbf{1}_{N_{\tau} \times 1} .
\end{gathered}
$$

where $\mathbf{1}_{N_{\tau} \times 1} \in \mathbb{R}^{N_{\tau} \times 1}$ and $\mathbf{1}_{1 \times N_{\text {out }}} \in \mathbb{R}^{1 \times N_{\text {out }}}$ are the oneentry vectors, $\mathbf{0}_{\left(N_{\tau} N_{\text {out }}\right) \times 1} \in \mathbb{R}^{\left(N_{\tau} N_{\text {out }}\right) \times 1}$ and $\mathbf{0}_{\left(N_{\tau} N\right) \times 1} \in$ $\mathbb{R}^{\left(N_{\tau} N\right) \times 1}$ are the zero-entry vectors, and $\mathbf{I}_{N_{\tau}} \in \mathbb{R}^{N_{\tau} \times N_{\tau}}$ is the identity matrix. Eq. 22a integrates feasibility conditions (8a) and (8b). Constraint equations 22b, 22c, and 22d are identical to $8 \mathrm{c}), 8 \mathrm{~d})$, and $8 \mathrm{e}$ respectively.

Theorem 2. If $u_{i}[k] \geq 0$ at every $i \in \mathcal{V}_{i n}, v_{j}[k] \geq 0$ at every $j \in \mathcal{V}_{\text {out }}, \rho_{i}[k]$ is updated by dynamics [2], and $\rho_{i}[0] \geq 0$ at every node $i \in \mathcal{V}_{I}$, then $\rho_{i}[k] \geq 0$ at every interior road element $i \in \mathcal{V}_{I}$ and every discrete time $k$.

Proof: By applying the mass conservation law in (2), traffic network dynamics can be obtained via Eq. (9b) at every interior road $i \in \mathcal{V}_{I}$. Per Assumption $11, p_{i}[k] \in[0,1)$ at every $i \in \mathcal{V}_{I}$ and all discrete times $k$, which indicate that $\left(1-p_{i}[k]\right)>0$ on the right-hand side of Eq.9b). Also, $q_{i, j}[k]$ is defined as a quantity in interval $[0,1]$ at every discrete sampling time $k$. If $\rho_{i}[0] \geq 0, u_{i}[k] \geq 0$ at every $i \in \mathcal{V}_{i n}$, and $v_{j}[k] \geq 0$ at every $j \in \mathcal{V}_{\text {out }}$, then the righthand side of Eq. (9b) must be a non-negative quantity at every discrete sampling time $k$. This implies that $\rho_{i}[k] \geq 0$ at every node $i \in \mathcal{V}_{I}$ and discrete time $k$.

Per Theorem 2. $\rho_{i}[k] \geq 0$ at every $\operatorname{road} i \in \mathcal{V}_{I}$ and discrete time $k$. Therefore, condition 22c) is redundant, and conditions (22a), 22b), and (22d) are sufficient to determine the feasible optimal boundary input $\mathbf{U}^{*}[k]$ by solving the following quadratic programming problem:

$$
\min \frac{1}{2}\left(\mathbf{U}[k]^{\top} \mathbf{U}[k]\right)
$$

subject to equality constraint 22d and inequality constraint

$$
\left[\begin{array}{c}
-\mathbf{I}_{N_{\tau} N_{\text {out }}} \\
\mathbf{H}[k]
\end{array}\right] \mathbf{U}[k] \leq\left[\begin{array}{c}
\mathbf{0}_{\left(N_{\tau} N_{\text {out }}\right) \times 1} \\
\mathbf{1}_{N_{\tau} \times 1} \otimes \mathbf{x}_{\max }-\mathbf{G}[k] \mathbf{x}[k]
\end{array}\right] .
$$

Note that

$$
\mathbf{s}^{*}[k]=\left[\begin{array}{ll}
\mathbf{I}_{N_{\text {out }}} & \left.\mathbf{0}_{N_{\text {out }} \times\left(\left(N_{\tau}-1\right) N_{\text {out }}\right)}\right]
\end{array} \mathbf{U}^{*}[k]\right.
$$

is the optimal boundary control at discrete time $k$.

\section{Simulation Results}

In this section, we present the simulation results of modeling and control in the example NOIR shown in Fig 1 . This particular NOIR consists of 259 road elements of Center City Philadelphia, where the index numbers of the road elements are shown in Fig, 1 . We process the map data generated by SUMO and obtain the corresponding graph $\mathcal{G}(\mathcal{V}, \mathcal{E})$. Node set $\mathcal{V}=\{1, \cdots, 259\}$ can be expressed as $\mathcal{V}=\mathcal{V}_{\text {in }} \cup \mathcal{V}_{\text {out }} \cup \mathcal{V}_{I}$ and $\mathcal{V}_{\text {in }}=\{1, \cdots, 20\}, \mathcal{V}_{\text {out }}=$ $\{21, \cdots, 42\}, \mathcal{V}_{I}=\{43, \cdots, 259\}$.

We set the whole simulation time as $3000 \mathrm{~s}$ and the sampling interval as $20 \mathrm{~s}$, which implies that the traffic coordination is simulated for 150 time steps. At every discrete time $k \in\{1, \cdots, 150\}$ the outflow probability matrix $\mathbf{P}[k]$ and the fraction probability matrix $\mathbf{Q}[k]$ are randomly generated to simulate the uncertainty of human driving intent roughly. For simulation, we choose $u_{0}=100$, which implies 100 cars are permitted to cross the boundary of the NOIR shown in Fig 1 at every discrete time $k$. For every $\operatorname{road} i \in \mathcal{V}_{I}$,

$$
\rho_{i, \text { max }}=\frac{n_{i, \text { lane }} * l_{i}}{l_{\text {veh }}}
$$

assigns an upper bound for the number of cars at road element $i \in \mathcal{V}_{I}$, where $l_{v e h}=4.5 \mathrm{~m}$ is considered the same for all road elements, $l_{i}$ is the length of road element $i \in \mathcal{V}_{I}$ in the Center City area, and $n_{i, \text { lane }}$ refers to the number of lanes at road element $i \in \mathcal{V}_{I}$. Meanwhile, the initial traffic density $\rho_{i}[0]$ is assigned randomly for every road element $i \in \mathcal{V}$.

We plot simulation results for two inlet boundary road elements $8,17 \in \mathcal{V}_{\text {in }}$, two outlet boundary road elements $27,35 \in \mathcal{V}_{\text {out }}$, and two interior road elements $68,119 \in \mathcal{V}_{I}$. The locations of these six road elements are presented in Table [1]

Fig. 2 plots the density variations versus discrete sampling time $k$ at the example interior road elements. It can be observed that the traffic density of road elements 68 and 119 
TABLE I: Example road elements in NOIR

\begin{tabular}{|l|l|l|}
\hline Type & Road Index & Name and Location \\
\hline Inlet & 8 & Cherry St. (N20th-N19th) \\
\hline Inlet & 17 & S11th St. (Locust- Walnut) \\
\hline Outlet & 27 & N16th St. (Race-Vine ) \\
\hline Outlet & 35 & Sansom St. (N20th-S19th) \\
\hline Interior & 68 & Filbert St. (N12th-N13th ) \\
\hline Interior & 119 & JFK Blvd (N16th-N17th) \\
\hline
\end{tabular}
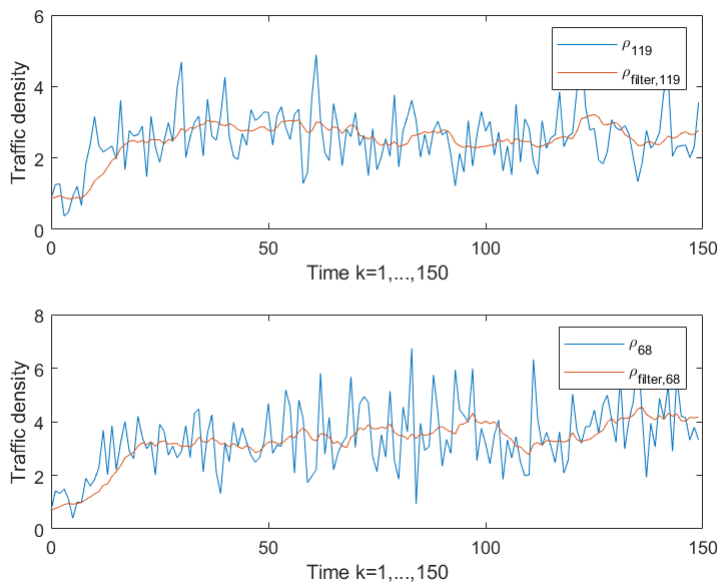

Fig. 2: Variation trends of traffic densities at example interior road elements

reaches the steady-state condition when the discrete sampling time $k>20$.

Fig. 3 illustrates the variation of the external traffic flow at inlet road elements $8,17 \in \mathcal{V}_{\text {in }}$ and outlet road elements $27,35 \in \mathcal{V}_{\text {out }}$. The variation trends at inlet and outlet road elements are similar: After a period of variation, the external traffic inflows and the external traffic outflows reach the steady state condition. Fig. 4 presents the net inflow and outflow of the NOIR versus discrete sampling time $k$. It could be observed that, after starting the simulation, the amount of traffic inflow decreases while the amount of traffic outflow increases symmetrically and after about 30 sampling times they converge to a stable state. We can formulate this variation trend as

when $k>30$.

$$
\sum_{i \in \mathcal{V}_{\text {in }}} u_{i}[k]=\sum_{i \in \mathcal{V}_{\text {out }}} v_{i}[k] \cong 50,
$$

\section{CONCLUSION}

This paper introduce a conservation-based modeling method to learn the traffic network dynamics and alleviate the traffic congestion. We apply the mass conservation law to model traffic coordination by a time-varying stochastic process where the real map data is used to define the traffic network. We offer an MPC control to manage traffic congestion by controlling the inflow and outflow at the boundary of the NOIR. The simulation results demonstrate that our proposed modeling and control approach can manage the traffic congestion effectively through optimizing the traffic inflow and outflow across the boundary of the NOIR. In our
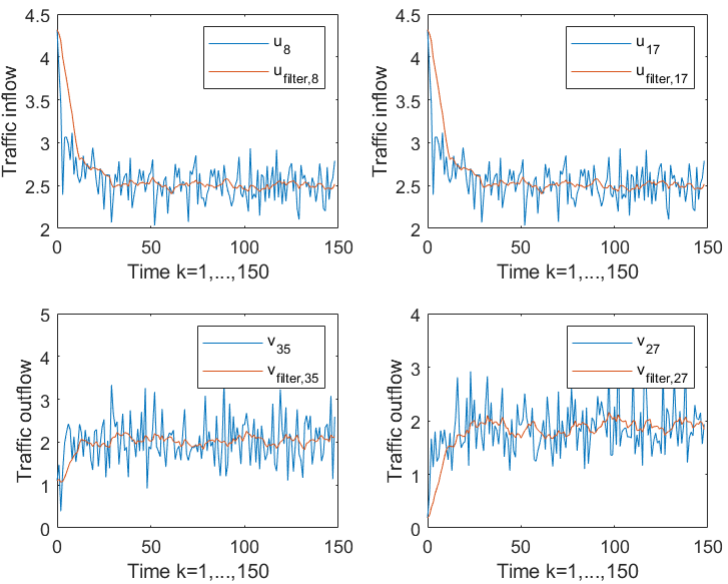

Fig. 3: External traffic flows at example inlet and outlet road elements

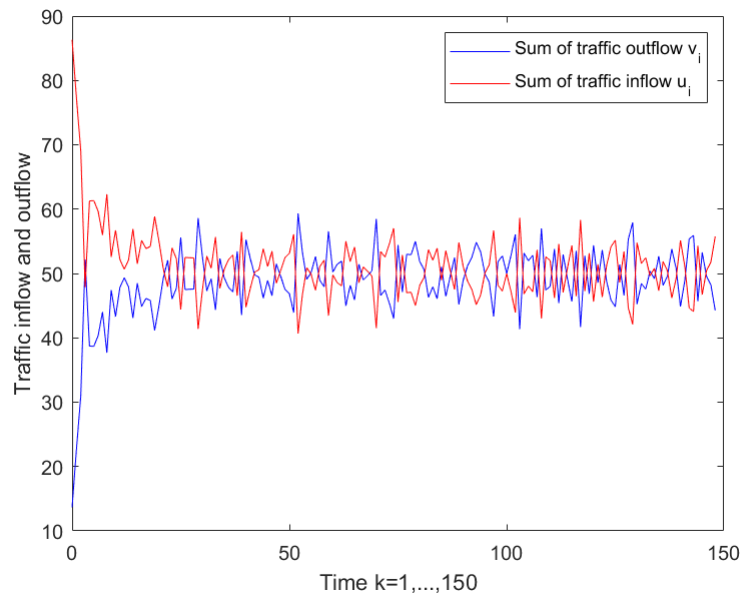

Fig. 4: External traffic inflow and outflow of the NOIR

future work, we plan to obtain the traffic dynamics based on real traffic data and control congestion through the boundary ramp meters and traffic signals, situated at road intersections.

\section{ACKNOWLEDGMENT}

The authors would like to acknowledge the Mechanical Engineering $\mathrm{PhD}$ fellowship provided to Xun Liu which was made possible by a generous gift from Dr. Yongping Gu and Fei Gu.

\section{REFERENCES}

[1] Muneera, C. P., and Karuppanagounder, K., 2018. "Economic impact of traffic congestion- estimation and challenges". European Transport / Trasporti Europei, 68

[2] O'Mahony, M., and Finlay, H., 2004. "Impact of traffic congestion on trade and strategies for mitigation". Transportation Research Board, 1873, pp. 25-34

[3] Ye, L., Hui, Y., and Yang, D., 2013. "Road traffic congestion measurement considering impacts on travelers". Journal of Modern Transportation, 21, pp. 28-39. 
[4] Annan, J., Mensah, J., and Boso, N., 2015. "Traffic congestion impact on energy consumption and workforce productivity:empirical evidence from a developing country". Archives of Business Research, 3, pp. 4054

[5] Chin, H., and Rahman, M., 2011. "An impact evaluation of traffic congestion on ecology". Planning Studies and Practice, 3, pp. 32-44.

[6] Zhang, X., Onieva, E., Perallos, A., Osaba, E., and Lee, V. C., 2014. "Hierarchical fuzzy rule-based system optimized with genetic algorithms for short term traffic congestion prediction". Transportation Research Part C, 43, pp. 127-142.

[7] Ito, T., and Kaneyasu, R., 2017. "Predicting traffic congestion using driver behavior". Procedia Computer Science, 112, pp. 1288-1297.

[8] Huang, Z., Xia, J., Li, F., Li, Z., and Li, Q., 2019. "A peak traffic congestion prediction method based on bus driving time". Entropy, 21, p. 709 .

[9] Kong, X., Xu, Z., Shen, G., Wang, J., Yang, Q., and Zhang, B., 2016. "Urban traffic congestion estimation and prediction based on floating car trajectory data". Future Generation Computer Systems, 61, pp. 97107.

[10] Tettamanti, T., Horváth, M. T., and Varga, I., 2017. "Nonlinear traffic modeling for urban road network and related robust state estimation". In 9th European Nonlinear Dynamics Conference (ENOC 2017), EUROMECH, p. 247.

[11] Geroliminis, N., and Daganzo, C. F., 2008. "Existence of urban-scale macroscopic fundamental diagrams: Some experimental findings". Transportation Research Part B: Methodological, 42(9), pp. 759 770.

[12] Xu, F., He, Z., Sha, Z., Sun, W., and Zhuang, L., 2013. "Traffic state evaluation based on macroscopic fundamental diagram of urban road network". Procedia Social and Behavioral Sciences, 96, pp. 480-489.

[13] Sirmatel, I. I., and Geroliminis, N., 2017. "Integration of perimeter control and route guidance in large-scale urban networks via model predictive control". In Transportation Research Board 96th Annual Meeting, Transportation Research Board, p. 13p.

[14] Li, Z., Jin, S., Xu, C., and Li, J., 2019. "Model-free adaptive predictive control for an urban road traffic network via perimeter control". IEEE Access, 7, 11, pp. 172489-172495.

[15] Yang, L., Yin, S., Han, K., Haddadc, J., and Hu, M., 2017. "Fundamental diagrams of airport surface traffic: Models and applications". Transportation Research Part B: Methodological, 106, pp. 29-51.

[16] Shao, P., Wang, L., Qian, W., Wang, Q.-G., and Yang, X.-H., 2018. "A distributed traffic control strategy based on cell-transmission model". IEEE Access, 6, pp. 10771-10778.

[17] Munoz, L., Sun, X., Horowitz, R., and Alvarez-Icaza, L., 2003. "Traffic density estimation with the cell transmission model". Vol. 5, pp. $3750-3755$.

[18] Yin, S., Yang, L., and Han, K., 2017. "Off-block flow optimisation based on cell transmission model". DASC.

[19] Feldman, O., and Maher, M., 2002. "A cell transmission model applied to the optimisation of traffic signals".

[20] Gregurić, M., Vujić, M., Alexopoulos, C., and Miletić, M., 2020. "Application of deep reinforcement learning in traffic signal control: An overview and impact of open traffic data". Applied Sciences, 10, 06, p. 4011.

[21] Lin, Y., Dai, X., Li, L., and Wang, F.-Y., 2018. An efficient deep reinforcement learning model for urban traffic control

[22] Abdulhai, B., Pringle, R., and Karakoulas, G., 2003. "Reinforcement learning for true adaptive traffic signal control". Journal of Transportation Engineering, 129, 05.

[23] Mannion, P., Duggan, J., and Howley, E., 2016. An Experimental Review of Reinforcement Learning Algorithms for Adaptive Traffic Signal Control. 05, pp. 47-66.

[24] L.A., P., and Bhatnagar, S., 2011. "Reinforcement learning with function approximation for traffic signal control". Intelligent Transportation Systems, IEEE Transactions on, 12, 07, pp. 412 - 421.

[25] Rastgoftar, H., and Atkins, E., 2019. An integrative data-driven physics-inspired approach to traffic congestion control.

[26] Rastgoftar, H., and Girard, A., 2020. "Resilient physics-based traffic congestion control". pp. 4120-4125.

[27] Rastgoftar, H., and Jeannin, J.-B., 2021. A physics-based finite-state abstraction for traffic congestion control.

[28] Lin, S., De Schutter, B., Xi, Y., and Hellendoorn, H., 2011. "Fast model predictive control for urban road networks via milp". Intelligent Transportation Systems, IEEE Transactions on, 12, 10, pp. 846 - 856. 